\title{
Escorpionismo em crianças e adolescentes: aspectos clínicos e epidemiológicos de pacientes hospitalizados
}

\author{
Scorpion poisoning among children and adolescents: clinical \\ and epidemiological characteristics of hospitalized patients
}

\author{
Fátima Maria Barbosa Horta ${ }^{1}$, Antônio Prates Caldeira ${ }^{1}$ \\ e Janer Aparecida S. Sares ${ }^{1}$
}

\begin{abstract}
RESUMO
Foram identificados 325 prontuários de crianças e adolescentes vítimas de picadas de escorpião (14,8\% de casos leves, 55,4\% de casos moderados e 29,8\% de casos graves). As variáveis associadas com maior gravidade foram: ausência de dor no local da picada, relato de sonolência à admissão e intervalo maior que três horas entre o acidente e o atendimento hospitalar.
\end{abstract}

Palavras-chaves: Escorpiões. Criança. Estudos retrospectivos. Análise multivariada.

\section{ABSTRACT}

Medical records relating to 325 children and adolescents who suffered scorpion stings were identified (14.8\% were mild cases, $55.4 \%$ were moderate cases and $29.8 \%$ were severe cases). The variables associated with greater severity were: lack of pain at the sting location, sleepiness reported at hospital admission and a time interval greater than three hours between the accident and hospital attendance.

Key-words: Scorpions. Child. Retrospective studies. Multivariate analysis.

As picadas de escorpião destacam-se entre os acidentes com animais peçonhentos na infância, devido às particularidades de vida desses artrópodes e as frequientes exposições das crianças. Nessa faixa etária, existe 0 risco de maior gravidade ${ }^{2} 317$. Além da dor, principal manifestação local, náuseas, vômitos, dor abdominal, sialorréia, arritmias cardíacas, hipertensão ou hipotensão, choque, edema agudo de pulmão, tremores e confusão mental estão entre os achados clínicos mais freqüentes ${ }^{114}$. 0 presente estudo teve como objetivo conhecer os aspectos clínicos e epidemiológicos dos acidentes escorpiônicos em crianças e adolescentes admitidos em um hospital de referência, buscando identificar as variáveis associadas com maior gravidade dos casos.

A área de referência deste estudo foi o município de Montes Claros, ao Norte do Estado de Minas Gerais, onde o Hospital Universitário Clemente de Faria é o único centro de referência para o atendimento de vítimas de animais peçonhentos. Todos os prontuários de crianças e adolescentes internados com 0 diagnóstico de escorpionismo, no período de janeiro de 1996 a dezembro de 2005, foram analisados. Antes da coleta de dados, obteve-se o consentimento expresso da direção do hospital para a análise dos prontuários, assegurando-se o sigilo das informações. 0 presente estudo foi avaliado e aprovado pelo Comitê de Ética em Pesquisa da Universidade Estadual de Montes Claros (Unimontes).

Foram identificados e avaliados 325 prontuários. A idade dos pacientes variou de dois meses a 15 anos, com mediana de 4,9 anos. Observou-se discreto predomínio dos casos no sexo masculino (53,5\%). Em relação às características dos acidentes, observouse que $38,8 \%(n=126)$ aconteceram no período decorrido entre 17 e 21 horas. 0 tempo decorrido entre a picada e a admissão hospitalar variou de uma a 36 horas, com mediana de três horas. Quanto ao local da picada, foram mais freqüentemente acometidos os membros inferiores (40\%) e os membros superiores (34,7\%). As principais manifestações locais e sistêmicas, no momento da admissão hospitalar e durante o período de hospitalização, foram associadas aos sistemas cardiovascular, nervoso e digestório. Na Tabela 1, são apresentadas as principais manifestações observadas no momento da internação.

1. Departamento de Saúde da Mulher e da Criança da Universidade Estadual de Montes Claros, Montes Claros, MG.

Endereço para correspondência: Dr. Antônio Prates Caldeira. Rua Monte Pascoal 225, Ibitutuna, 39401-347 Montes Claros, MG.

Tel: 5538 3222-3879

e-mail: antonio.caldeira@unimontes.br

Recebido para publicação em 23/11/2006

Aceito em 8/2/2007 
Tabela 1 - Principais manifestações locais e sistêmicas observadas à admissão hospitalar em crianças e adolescentes vítimas de picadas de escorpião, Hospital Universitário Clemente de Farias, Montes Claros (MG), 1996-2005.

\begin{tabular}{lcc}
\hline Manifestações & Frequiência (n) & Percentual (\%) \\
\hline Locais & 297 & \\
dor & 127 & 91,4 \\
sudorese & 83 & 39,1 \\
eritema & 46 & 25,5 \\
edema & 21 & 14,2 \\
parestesia & 7 & 6,5 \\
equimose & & 2,2 \\
Sistêmicas & 285 & \\
náuseas/vômitos & 254 & 87,5 \\
taquicardia & 203 & 78,2 \\
agitação & 71 & 62,5 \\
tosse com sibilos/crepitações & 31 & 23,1 \\
sonolência & 23 & 9,5 \\
dor abdominal & 18 & 7,1 \\
sialorréia & & 5,5 \\
\hline
\end{tabular}

0 período de hospitalização variou de um a 18 dias, com uma média de 2,3 dias e o desfecho final foi satisfatório para 315 pacientes $(96,9 \%)$, que apresentaram alta hospitalar sem seqüelas. Para cinco pacientes (1,5\%) não foi possível verificar o desfecho final, pois foram transferidos para outras unidades hospitalares. Foram identificados cinco casos que evoluíram para o óbito após a internação hospitalar. A classificação final dos casos apresentou a seguinte proporção: $14,8 \%$ de casos leves $(n=48), 55,4 \%$ de casos moderados $(n=180)$ e $29,8 \%$ de casos graves $(n=97)$.

Com o objetivo de apontar variáveis capazes de predizer potencial gravidade do caso desde o momento da hospitalização, estudou-se a associação entre gravidade do caso e variáveis demográficas e clínicas à admissão. A Tabela 2 apresenta a primeira fase dessa análise. As variáveis que mostraram associação até 0 nível de significância de $20 \%(\mathrm{p}<0,20)$ foram avaliadas através de análise multivariada, que mostrou que, em uma análise conjunta, os fatores que se mostram estatisticamente associados com maior gravidade foram: ausência de dor local $(\mathrm{OR}=0,250$; IC95\%=0,086-0,724), o relato de sonolência no momento da admissão $(0 R=3,878$; IC95\%=1,584-9,493) e um atraso superior a três horas desde o momento da picada até a assistência hospitalar $(\mathrm{OR}=2,220$; IC95\%=1,235-4,054).

Os resultados apontados apresentam a limitação da análise de dados secundários e da coleta retrospectiva, todavia, são similares aos resultados apontados por outros autores ${ }^{3715}$. A faixa etária mais acometida (menores de cinco anos) é semelhante àquela apontada por estudo realizado na Venezuela ${ }^{15}$. As características clínicas e os locais mais acometidos apontados pela referida pesquisa também são semelhantes aos achados do presente estudo. 0 horário em que mais comumente se registrou 0 acidente que culminou com a internação hospitalar (final do dia e início da noite) é compatível com o horário em que os escorpiões habitualmente saem para buscar alimentos ${ }^{14}$.
Tabela 2 - Fatores associados com maior gravidade de casos de escorpionismo em crianças e adolescentes hospitalizados no Hospital Universitário Clemente de Farias (análise univariada); Montes Claros (MG), 1996-2005.

\begin{tabular}{|c|c|c|c|c|}
\hline \multirow[b]{2}{*}{ Variável } & \multicolumn{2}{|c|}{ Gravidade } & \multirow[b]{2}{*}{$\mathrm{p}$} & \multirow[b]{2}{*}{ OR (IC95\%) } \\
\hline & sim & $\overline{\text { não }}$ & & \\
\hline \multicolumn{5}{|l|}{ Sexo } \\
\hline masculino & 62 & 112 & 0,020 & $1,83(1,09-3,10)$ \\
\hline feminino & 35 & 116 & & \\
\hline \multicolumn{5}{|l|}{ Idade (anos) } \\
\hline$\leq 2$ & 20 & 44 & 0,903 & $1,09(0,57-2,05)$ \\
\hline$>2$ & 77 & 184 & & \\
\hline \multicolumn{5}{|l|}{ Procedência } \\
\hline outras cidades (zona rural) & 74 & 110 & 0,000 & $3,45(1,95-6,15)$ \\
\hline Montes Claros (zona urbana) & 23 & 118 & & \\
\hline \multicolumn{5}{|l|}{ Dor local } \\
\hline $\operatorname{sim}$ & 79 & 218 & 0,000 & $0,20(0,08-0,49)$ \\
\hline não & 18 & 10 & & \\
\hline \multicolumn{5}{|l|}{ Edema local } \\
\hline $\operatorname{sim}$ & 13 & 33 & 0,936 & $0,91(0,43-1,93)$ \\
\hline não & 84 & 195 & & \\
\hline \multicolumn{5}{|l|}{ Eritema local } \\
\hline $\operatorname{sim}$ & 23 & 60 & 0,724 & $0,87(0,48-1,57)$ \\
\hline não & 74 & 168 & & \\
\hline \multicolumn{5}{|l|}{ Parestesia local } \\
\hline $\operatorname{sim}$ & 3 & 18 & 0,172 & $0,37(0,08-1,40)$ \\
\hline não & 94 & 210 & & \\
\hline \multicolumn{5}{|l|}{ Sudorese local } \\
\hline $\operatorname{sim}$ & 40 & 87 & 0,692 & $1,14(0,68-1,91)$ \\
\hline não & 57 & 141 & & \\
\hline \multicolumn{5}{|l|}{ Náuseas/vômitos } \\
\hline $\operatorname{sim}$ & 88 & 197 & 0,368 & $1,54(0,66-3,67)$ \\
\hline não & 9 & 31 & & \\
\hline \multicolumn{5}{|l|}{ Sonolência } \\
\hline $\operatorname{sim}$ & 17 & 14 & 0,002 & $3,25(1,43-7,41)$ \\
\hline não & 80 & 214 & & \\
\hline \multicolumn{5}{|l|}{ Dor abdominal } \\
\hline $\operatorname{sim}$ & 8 & 15 & 0,768 & $1,28(0,47-3,38)$ \\
\hline não & 89 & 213 & & \\
\hline \multicolumn{5}{|l|}{ Taquicardia } \\
\hline $\operatorname{sim}$ & 86 & 168 & 0,004 & $2,79(1,33-5,99)$ \\
\hline não & 11 & 60 & & \\
\hline \multicolumn{5}{|l|}{ Tempo até assistência } \\
\hline até 3 horas & 54 & 80 & 0,000 & $2,70(1,57-4,65)$ \\
\hline após 3 horas & 35 & 140 & & \\
\hline
\end{tabular}

$\mathrm{p}=$ nível de significância estatística $<5 \%$; OR: odds ratio

As características clínicas dos casos analisados, tanto em relação às manifestações locais quanto sistêmicas são semelhantes a outros estudos nacionais ${ }^{2} 811$ e internacionais ${ }^{10}{ }^{17}$, com destaque para as manifestações neurológicas e cardiovasculares. Todavia, a letalidade observada para o grupo estudado (1,5\%) é bastante significativa, considerando a efetividade do soro específico e a informação do documento oficial do Ministério da Saúde, que destaca que a letalidade para os casos graves como inferior a $0,6 \%{ }^{6}$. A pesquisa nos prontuários dos pacientes não permitiu a identificação da espécie de escorpião mais associada com os acidentes, mas outros estudos já apontaram que as espécies 
mais comuns na região sudeste são Tityus bahiensis e Tityus serrulatus $^{12}{ }^{16}$, sendo essa última a que mais está associada com casos graves e letais $^{212}$.

Um aspecto relevante do presente estudo foi a busca por elementos capazes de predizer maior gravidade a partir do exame inicial da criança ou adolescente. A análise estatística final mostrou que as variáveis do exame inicial que estiveram associadas com maior gravidade foram: a ausência de dor local, a presença de sonolência no momento da admissão e um tempo maior do que três horas decorrido entre a picada e a admissão hospitalar.

A presença de dor local é extremamente comum após a picada do escorpião. Ela decorre, provavelmente, de ação direta do veneno, através de proteínas neurotóxicas ${ }^{14}$. Em princípio, a ausência de dor local como um fator associado a maior gravidade não parece ser lógico dentro da cadeia de eventos desencadeada pelo veneno do escorpião. Na avaliação dos autores, a ausência da dor pode estar associada com a presença de outras manifestações mais evidentes do paciente, que a tornariam um aspecto menos destacado pelo mesmo ou pelos profissionais assistentes (que não a registraram no prontuário).

A associação entre sonolência no momento da admissão e maior gravidade denota a ação de proteínas do veneno em sistema nervoso central, contudo esta é uma manifestação pouco destacada na literatura em geral ${ }^{3}$. Entre as várias manifestações neurológicas registradas após picadas de escorpião, existem relatos de dismetria, ataxia de marcha e mioclonias ${ }^{11}$, contraturas musculares $^{589}$ e disartria ${ }^{10}$. A pesquisa de tais manifestações na população pediátrica é comprometida pelas características próprias dessa faixa etária. Assim a sonolência se apresenta, para essa população, como uma das manifestações mais evidentes de comprometimento neurológico.

Finalmente, em relação à outra variável identificada pela análise multivariada, tempo superior a três horas para a admissão hospitalar, esta decorre do atraso na administração do soro específico. Embora alguns autores apresentem algumas dúvidas sobre a efetividade da soroterapia específica em casos graves ${ }^{2}$, a literatura recente apresenta dados incontestes sobre a necessidade e administração do soro anti-escorpiônico como forma de evitar desfechos indesejáveis ${ }^{4}{ }^{13}$. Seguramente, intervenções rápidas e eficazes contribuíram para que a letalidade observada no presente estudo não fosse maior. Além da necessidade de orientação para a população em geral, sobre a busca imediata ao serviço de referência e atividades educativas sobre os hábitos de vida dos escorpiões, faz-se necessária uma maior descentralização na distribuição do soro específico, com capacitação aos profissionais de saúde sobre adequado manejo clínico dos pacientes.

Concluindo, o presente estudo apresenta uma significativa casuística do escorpionismo em crianças e adolescentes, pois existem poucos estudos similares. É necessário que novos estudos sejam realizados para confronto das variáveis aqui apresentadas como associadas a maior gravidade do escorpionismo na infância, o que implica, necessariamente, em maiores riscos para a vítima e maiores custos hospitalares. Na verdade, enquanto o acidente escorpiônico se mantiver como um importante problema de saúde pública, existe uma necessidade ética de novos estudos que busquem maior compreensão do comportamento clínico e epidemiológico de tais eventos.

\section{AGRADECIMENTOS}

Os pesquisadores agradecem a contribuição dos estudantes Cristiane Leal Viana, Fernanda Angeli de Freitas, Isabela Maciel Fernandes e Pedro Henrique de Oliveira Almeida, pela colaboração durante a coleta de dados.

\section{REFERÊNCIAS}

1. Amitai Y. Clinical manifestation and management of scorpion envenomation. Public Health Reviews 26:257-263, 1998.

2. Bucaretchi F, Baracat ECE, Nogueira RJN, Chaves A, Zambrone FAD, Fonseca MRCC, Tourinho FS. A comparative study of severe scorpion envenomation in children caused by Tityus babiensis and Tityus serrulatus. Revista do Instituto de Medicina Tropical de São Paulo 37:331-336, 1995.

3. Campos JA, Costa DM, Oliveira JS. Acidentes por animais peçonhentos. In: Tonelli E, Freire LMS (eds) Doenças infecciosas na infância e adolescência, $2^{a}$ edição, Medsi Editora, p.1531-1556, 2000.

4. Chowell G, Diaz-Dueñas P, Bustos-Saldaña R, Mireles AA, Fet V. Epidemiological and clinical characteristics of scorpionism in Colima, México (2000-2001). Toxicon 47:753-758, 2006

5. Cupo P, Jurca M, Azevedo-Marques MM, Oliveira JSM, Hering SE. Severe scorpion envenomation in Brazil: Clinical, laboratory and anatomicopathological aspects. Revista do Instituto de Medicina Tropical de São Paulo 36:67-76, 1994.

6. Fundação Nacional de Saúde. Manual de Diagnóstico e Tratamento de Acidentes por Animais Peçonhentos. Ministério da Saúde, Brasília, p. 141, 1998.

7. Gordillo ME. Escorpionismo en Pediatría. Archivos Argentino de Pediatría 98:296303,2000 .

8. Lira-da-Silva RM, Amorim AM, Brazil TK. Envenenamento por Tityus stigmurus (Scorpiones; Buthidae) no Estado da Bahia, Brasil. Revista da Sociedade Brasileira de Medicina Tropical 33:239-245, 2000.

9. Montoya-Cabrera MA. Alacranismo. Gaceta Médica de México 132:645-648, 1996.

10. Müller GJ. Scorpionism in South Africa. A report of 42 serious scorpion envenomations. South African Medical Journal 83:405-411, 1993.

11. Pardal PPO, Castro LC, Jennings E, Pardal JSO, Monteiro MRCC. Aspectos epidemiológicos e clínicos do escorpionismo na região de Santarém, Estado do Pará, Brasil. Revista da Sociedade Brasileira de Medicina Tropical 36:349-353, 2003.

12. Ribeiro AL, Rodrigues L, Jorge MT. Aspectos clínicos e epidemiológicos do envenenamento por escorpiões em São Paulo e municípios próximos. Revista de Patologia Tropical 30:83-92, 2001.

13. Riley BD, LoVecchio F, Pizon AF. Lack of scorpion antivenom leads to increased pediatric ICU admissions. Annals of Emergency Medicine 47:398-399, 2006.

14. Saldarriaga CM, Otero PR. Los escorpiones: aspectos ecológicos, biológicos y toxinológicos. MedUNAB 3:17-23, 2000.

15. Sequera L, Sandoval L, Chávez A. Emponzoñamiento en niños por escorpión tityus discrepans. Archivos Venezolanos de Puericultura y Pediatría 56:44-47, 1993.

16. Soares MRM, Azevedo CS, De Maria M. Escorpionismo em Belo Horizonte, MG: um estudo retrospectivo. Revista da Sociedade Brasileira de Medicina Tropical 35:359-363, 2002

17. Tamassone R, Vainstub V, Peirano S. Envenenamiento grave por escorpión en Pediatría. Archivos Argentino de Pediatría 101:392-397, 2003. 